\title{
Política de humanização (HumanizaSUS): uma política transversal na saúde
}

\author{
Humanization policy (HumanizaSUS): a transversal health policy
}

Política de humanización (HumanizaSUS): una política transversal en la salud

Simone Lopes de Almeida ${ }^{1 *}$, Calvino Camargo ${ }^{1}$, Kristiane Alves Araújo ${ }^{1}$ Ana Paula Barbosa Alves ${ }^{2,}$ Hosana Carolina dos Santos Barreto ${ }^{2}$.

\section{RESUMO}

Objetivo: Realizar revisão sistemática acerca das contribuições da Política Nacional de Humanização do Sistema único de Saúde (HumanizaSUS) enquanto política transversal na saúde a partir de seus métodos, diretrizes e dispositivos. Métodos: Trata-se de uma revisão bibliográfica, de artigos da base Scielo, publicados a partir do ano 2000 à atualidade a partir dos descritores: Humanização, Política de saúde e Sistema Único de Saúde. Revisão Bibliográfica: Dentro da perspectiva do HumanizaSUS, a saúde não deve ser realizada apenas no âmbito do sistema de saúde ou instituições, mas deve contemplar também o cuidado em saúde. Humanizar a atenção e a gestão em saúde se configura como meio para a qualificação das práticas de saúde através da ampliação do acesso com acolhimento; valorização dos trabalhadores e usuários; atenção integral com responsabilização e vínculo; avanço da gestão participativa e envolvimento direto do controle social. Considerações finais: $O$ estudo proposto atesta a partir de vários autores a relevância da Política de Humanização enquanto Política transversal do SUS, uma vez que sua proposta ética, estética e política, requer quebra de paradigmas concernentes às relações verticalizadas entre os sujeitos representativos da saúde, os colocando em relações horizontais e coletivas.

Palavras-chave: Humanização, Política de Saúde, Sistema Único de Saúde.

\section{ABSTRACT}

Objective: To conduct a systematic review of the contributions of the National Humanization Policy of the Single Health System (HumanizaSUS) as a cross-cutting policy in health from its methods, guidelines and devices. Methods: This is a bibliographic review, of articles from the Scielo database, published from the year 2000 to the present day, based on the descriptors: Humanization, Health Policy and the Unified Health System. Bibliographic review: From the perspective of HumanizaSUS, health should not be performed only within the health system or institutions, but should also include health care. Humanizing health care and management is a means for the qualification of health practices through the expansion of access with hospitality; valuing workers and users; full attention with responsibility and attachment; advancement of participatory management and direct involvement of social control. Final considerations: The proposed study attests from several authors the relevance of the Humanization Policy as a transversal SUS policy, since its ethical proposal, aesthetic and political, requires break of paradigms concerning the vertical relations between the subjects.

Kewords: Humanization, Health Policy, Unified Health System.

${ }^{1}$ Docente do curso de Medicina da Universidade Federal de Roraima (UFRR). Boa Vista-RR.

2 Docente do Instituto de Formação Superior Indígena da Universidade Federal de Roraima (UFRR). Boa

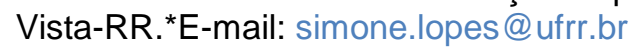




\section{RESUMEN}

Objetivo: Realizar una revisión sistemática de las contribuciones de la Política Nacional de Humanización del Sistema Único de Salud (HumanizaSUS) como una política transversal en materia de salud a partir de sus métodos, directrices y dispositivos. Métodos: Esta es una revisión bibliográfica, de artículos de la base de datos Scielo, publicados desde el año 2000 hasta la actualidad, basados en los descriptores: Humanización, Política de Salud y el Sistema Único de Salud. Revisión bibliográfica: desde la perspectiva de HumanizaSUS, la salud no debe realizarse solo dentro del sistema o las instituciones de salud, sino que también debe incluir la atención médica. La humanización de la atención y la gestión de la salud es un medio para la calificación de las prácticas de salud a través de la expansión del acceso con hospitalidad; la valoración de trabajadores y usuarios; Atención plena con responsabilidad y apego; Avance de la gestión participativa y participación directa del control social. Consideraciones finales: El estudio propuesto atestigua a varios autores la relevancia de la Política de Humanización como una política transversal del SUS, ya que su propuesta ética, estética y política, requiere romper los paradigmas relacionados con las relaciones verticales entre los sujetos.

Palabras clave: Humanización, Política de salud, Sistema de Salud Unificado.

\section{INTRODUÇÃO}

O direito à saúde instituído a partir da criação do Sistema Único de Saúde (SUS) pela lei 8.080/90, que dispõe sobre as condições para a promoção, proteção e recuperação da saúde, a organização e o funcionamento dos serviços de saúde no Brasil, enfrenta dificuldades de cumprir seu papel de forma efetiva, uma vez que princípios como acesso, integralidade e financiamento ainda são grandes desafios (BRASIL, 1990).

No SUS, as ações de saúde integram uma rede regionalizada e hierarquizada segundo a sua complexidade (atenção primária, secundária e terciária), requerendo mudanças no modo de planejar, organizar e gerir as ações e serviços de saúde (ALMEIDA ND, 2013).

O sistema de saúde brasileiro configura-se uma das maiores conquistas dos cidadãos e traz como um dos seus princípios a participação da comunidade como estratégia de democratização da saúde. Tal gestão, dentro da perspectiva da Política Nacional de Humanização (PNH), não deve ser realizada apenas no âmbito do sistema de saúde ou instituições, mas deve contemplar também o cuidado em saúde (ALMEIDA SL, 2013).

A Política Nacional de Humanização da Atenção e Gestão no SUS (PNH) é uma política do SUS, também chamada de HumanizaSUS, traçada a partir de princípios e diretrizes, cuja operacionalização se dá por meio de métodos e dispositivos pautados na tríplice inclusão de sujeitos representativos na saúde: usuários, gestores e trabalhadores (SANTOS FILHO SB 2009; BRASIL, 2004, 2007), almejando mudanças e transformações na atenção e gestão dos serviços de saúde brasileiro.

Portanto, o presente artigo parte da premissa de buscar saber qual a contribuição da PNH enquanto política de saúde, para os gestores, profissionais de saúde e usuários? Nesse sentido, o presente estudo tem como objetivo realizar revisão sistemática e reflexiva acerca da Política Nacional de Humanização do SUS (HumanizaSUS) e suas contribuições enquanto política transversal na saúde a partir de seus métodos, diretrizes e dispositivos na dinâmica do SUS efetivo, equitativo e de qualidade.

\section{MÉTODOS}

Trata-se de um estudo bibliográfico cujo percurso metodológico percorrido sustentou-se em leituras seletivas relacionadas ao objeto de pesquisa, bem como em sua revisão integrativa, contribuindo assim para a síntese e análise dos resultados de vários estudos, concebendo um corpo de literatura coerente. 
Utilizou-se o estudo bibliográfico a partir de consultas a artigos da base Scientific Electronic Library Online (SCIELO), na língua portuguesa, documentos oficiais do Ministério da Saúde e legislações, de forma a respaldar a proposta da pesquisa. Sendo utilizados os descritores: Humanização, Política de Saúde e Sistema Único de Saúde.

Os critérios de inclusão foram: artigos publicados na Biblioteca eletrônica SciELO, a partir dos anos de 2000 à atualidade, artigos originais e publicados no Brasil, quanto à manuais e legislações específicas da saúde, incluíram-se exclusivamente os de Políticas e Programas de Saúde nacional. Os critérios de exclusão: artigos não indexados na Scielo, de origem internacional, políticas de saúde e portarias de outros países, teses e dissertações.

\section{RESULTADOS e DISCUSSÃO}

\section{Política de humanização da saúde: uma política transversal}

O termo humanização é amplamente utilizado nas diversas áreas de conhecimento tais como as Ciências Humanas, Exatas, Biológicas, da Saúde e Ciências Sociais, dentre outras, que trazem variadas abordagens e vertentes que a norteia conforme o seu interesse técnico-científico e social (RIOS IC, 2009).

Rios IC (2009) assevera que o termo humanização tem sua gênese a partir de preceitos filosóficos pautados no Humanismo, que reconhece o valor do homem a partir da sua essência, incluindo-se suas potencialidades, limites e interesses. O Humanismo busca entender o homem e engendrar mecanismos que os façam compreender-se uns aos outros.

O conceito de humanização surge como um princípio vinculado ao paradigma de direitos humanos expressos individual e socialmente, sendo inicialmente contemplado na Declaração Universal dos Direitos do Homem, que se funda na noção de dignidade e igualdade de todos os seres humanos. Assim, o núcleo do conceito de humanização está intrinsecamente interligado a noção de dignidade e respeito à vida, enfatizando-se a dimensão ética na relação entre pacientes e profissionais de saúde. Esta noção começou a ser mais amplamente utilizada na área da saúde a partir dos anos 90, quando vários países membros da Organização Mundial da Saúde (OMS) assinaram declarações relativas a direitos de pacientes (VAISTMAN J e ANDRADE GRB,2005).

Porém, o termo humanização na saúde transcende essa proposta, sendo além de uma causa humanitária e de direito à saúde, a visão ampliada da gestão e do cuidado, onde são principalmente incluídos a defesa da vida (BRASIL, 2007).

Sobre o começo das discussões sobre a humanização na saúde no Brasil, Rios IC (2009) explica que a mesma se originou juntamente com o SUS, cujos princípios da universalidade, integralidade, equidade e participação da comunidade são de inspiração humanista, o que torna o SUS atualmente como o principal sistema de inclusão social de nosso país.

O SUS configura-se como o modelo público de ações e serviços de saúde norteado por um conjunto de princípios e diretrizes únicas para todo o território nacional, de forma a articular ações de promoção da saúde, prevenção de doenças e reabilitação da saúde em todos os níveis de complexidade da assistência, seja baixa, média ou alta complexidade em todas as esferas de governo: Municipal, Estadual e Federal (BRASIL, 1990, 2013; SILVA JUNIOR DN ,et al. 2018).

Para Deslandes SF (2004), a temática da humanização na saúde brasileira vem sendo utilizada há décadas nas pautas dos movimentos feministas, na busca da humanização do parto e do respeito aos direitos reprodutivos das mulheres, já para as crianças fez parte de um escopo mais direcionado ao atendimento de bebês de baixo peso, internados em UTI.

Assevera-se que timidamente este tema se anuncia desde a XI Conferência Nacional de Saúde (CNS, 2000), que tinha como título: Acesso, qualidade e humanização na atenção à saúde com controle social, procurando interferir nas agendas das políticas públicas de saúde. (BENEVIDES R e PASSOS E, 2005). 
Tal fato atesta que a quase duas décadas o tema contempla a agenda da discussão dos sujeitos representativos, uma vez que o controle social do SUS, representado pelos Conselhos e Conferências de Saúde, já incluía a Humanização em suas pautas.

Pasche DF (2009) assegura que a participação cidadã em saúde é espaço de abertura para a construção, com o conjunto da sociedade, de processos de corresponsabilização na gestão da política de saúde, sem com isto desresponsabilizar o Estado de suas funções essenciais.

Tais espaços coletivos e arenas decisórias na formulação e gestão de políticas públicas, configura-se uma estratégia importante de democratização do Estado e do acesso aos serviços de saúde.

O conceito de humanização foi estabelecido a princípio, a partir de dois programas de saúde no setor público brasileiro, o Programa de Humanização da Assistência Hospitalar (PNHAH), criado através da Portaria no 881, de 19 de de junho 2001 e o Programa de Humanização no Pré-Natal e Nascimento (PHPN), criado através da Portaria ํㅡ 569/GM, em 1ํ de junho de 2000.Porém ambas se limitavam à área hospitalar.

Percebe-se que inicialmente, a mesma foi concebida como Programa de saúde, direcionado a um público restrito: mulheres e crianças, institucionalizadas em Hospitais. No ano de 2003, o Ministério da Saúde criou a Política Nacional de Humanização (PNH) a partir da revisão da PNHAH, ampliando a proposta da humanização para além dos hospitais de forma a contemplar toda a rede SUS e estabeleceu uma política focada nos processos de trabalho e de gestão da saúde (RIOS IC, 2009).

Sobre isso Campos GWS (2005) acrescenta que no campo das políticas de saúde a humanização voltase para as práticas concretas comprometidas com a produção de sujeitos de tal modo que atender melhor o usuário se dá em sintonia com melhores condições de trabalho e de participação dos diferentes sujeitos implicados no processo de produção de saúde.

Tal pensamento reforça que a PNH implica em mudança nos processos de trabalho, atendimento de necessidade e melhor satisfação dos sujeitos envolvidos na produção da saúde, seja ele gestor, trabalhador ou usuário.

Ferreira LR e Artman E (2018) asseguram que a Política de Humanização reafirma a necessidade de se investir na qualidade do cuidado, e não apenas na expansão da rede e do acesso, pois deve considerar o respeito ao usuário e dignidade do trabalhador da saúde. Fato explícitio na tríplice inclusão: usuários, trabalhadores e gestores.

Enquanto política, a PNH chamada atualmente de Política Nacional de Humanização da Gestão e da Atenção do SUS se estrutura em diretrizes, métodos, dispositivos e princípios, onde Rios IC (2009) comenta que tais diretrizes apontam como caminhos:fortalecimento dos trabalhos em equipe, cuja transdisciplinaridade e grupalidade permeiam as relações; a promoção do cuidado ao usuário e o trabalhador; até a dileção das práticas de atenção e gestão em sua essência e subjetividades, fato que promove o fortalecimento de compromissos e responsabilidades conjuntas; bem como a utilização da comunicação, educação permanente, informação e dos lócus da gestão na construção de autonomia e protagonismo dos sujeitos envolvidos.

Falar em protagonismo dos sujeitos é ressaltar a importância da tríplice inclusão, pois os coloca em relações horizontais e coletivas, cujos problemas e desafios são enfrentados de forma compartilhada (CAMPOS GWS, 2005).

Portanto, uma diretriz ética e política que visa motivar e educar os trabalhadores. Ou seja, um modelo de gestão, centrado no trabalho em equipe, na construção coletiva e em colegiados que garantem que o poder seja de fato compartilhado, por meio de análises, decisões e avaliações construídas coletivamente (BRASIL, 2004). Dessa forma, busca promover saúde nos locais de trabalho a partir do aprimoramento da capacidade de compreensão e análise do trabalho pelo próprio trabalhador, de forma a fazer circular a palavra criando espaços para debates coletivos. A gestão coletiva das situações de trabalho é critério fundamental para a promoção de saúde. 
Brasil (2010) refere que a PNH possui três princípios norteadores a partir dos quais se desdobra enquanto política pública transversal de saúde que são: Transversalidade, Indissociabilidade entre atenção e gestão e Protagonismo, coresponsabilidade e autonomia dos sujeitos e dos coletivos. Sendo que tais princípios são as molas propulsoras da política.

A palavra Transversalidade deriva de Transversal, que segundo Ferreira ABH (2010) significa "Linha que corta ou que atravessa outra linha ou um plano", sendo enquanto princípio da PNH a proposta de perpassar todas as políticas, instâncias e sujeitos representativos no SUS.

No que se refere a Indissociabilidade entre atenção e gestão, a PNH (2013) refere que as decisões da gestão interferem diretamente na atenção à saúde. Por isso, trabalhadores e usuários devem buscar conhecer como funciona a gestão dos serviços e da rede de saúde, assim como participar ativamente do processo de tomada de decisão nas organizações de saúde e nas ações de saúde coletiva.

Tal princípio amplia-se ao cuidado em saúde, cuja responsabilidade não passa a ser apenas do profissional de saúde, mas também do gestor e do usuário enquanto sujeito protagonista da sua saúde.

Portanto, a proposta da PNH não está restrita aos serviços de prestação direta de assistência à saúde, como hospitais, outras unidades de saúde e à estratégia Saúde da Família (ESF). É também adequado ao âmbito das instâncias gestoras vinculadas ao SUS estadual e municipal (distritos sanitários, secretarias municipais e estaduais de saúde), do Ministério da Saúde e, também, das parcerias entre municípios e cooperações interinstitucionais (entidades formadoras, conselhos profissionais, etc.). (BRASIL, 2010a).

A proposta da PNH é ambiciosa e requer muita dedicação, que suplanta os interesses dos gestores, profissionais de saúde e usuários do SUS, esteja em uma metrópole ou em um recôndito município no interior da Amazônia. Esteja ele, em um Hospital de atendimento de alta complexidade ou em uma Unidade Básica de Saúde instalado em uma vila remota na margem de um rio na Amazônia. O que vai fazer o diferencial é o espírito de equipe, o sentido da inclusão, a gestão compartilhada, a mudança nos processos de trabalho, mudanças nas práticas de atenção e gestão, que geram satisfação em todos os sujeitos envolvidos, refletindo em uma saúde de qualidade e compatível com os princípios do SUS.

Percebe-se que a implementação da PNH, não é realizada através de ações momentâneas ou de esforços limitados e dispersos, mas é fruto de um trabalho integrado subsidiado por um plano sustentado, de forma contínua, complexa, permanente e multiforme, que envolve os mais diversos e variados níveis de responsabilidades, de modo a incluí-la como política prioritária de reafirmação e acessibilidade baseada transversalidade e na tríplice inclusão e gestão participativa, de um SUS que dá certo: universal, equitativo, integral e resolutiva.

No momento em que a Política de Humanização dos serviços de saúde adquire destaque dentro do cenário político, torna-se premente importante avaliar o grau de compreensão do processo, pelos gestores e profissionais que estão na linha de frente dos serviços de saúde e usuários do SUS, para que se possam realizar os devidos esclarecimentos e dirimir as dúvidas existentes, não permitindo que a mesma se torne apenas uma Política de saúde idealizada e não aplicada.

\section{CONSIDERAÇÕES FINAIS}

O estudo atesta a partir de vários autores a relevância da Política de Humanização enquanto política transversal do SUS, uma vez que sua proposta ética, estética e política, requer quebra de paradigmas concernentes às relações verticalizadas entre os sujeitos representativos da saúde, os colocando em relações horizontais e coletivas. Verifica-se a pertinência do tema em pauta no meio acadêmico, cursos de formação de movimentos sociais ou outros espaços de debates, buscando fomentar conhecimentos e reflexões acerca da sua magnitude frente a real aplicabilidade do HumanizaSUS nos serviços de saúde, cuja proposta de transformação dos processos de trabalho/relações/cuidados mais efetivos aspiram atender aos princípios fundamentais do SUS. 


\section{REFERÊNCIAS}

1. ALMEIDA ND. A saúde no Brasil, impasses e desafios enfrentados pelo Sistema Único de Saúde: SUS. Rev. Psicol. Saúde, 2013; 5(1):01-09.

2. ALMEIDA SL. Gestão da Saúde e Política de Humanização na Amazônia Brasileira. 2013. Dissertação (Mestrado em Ciências da Saúde) PROCISA. Universidade Federal de Roraima, Boa Vista, 2013; 115 p.

3. BENEVIDES R, PASSOS E. A humanização como dimensão pública das políticas de saúde. Ciência e Saúde Coletiva, 2005;10(3):561-571.

4. BRASIL. Lei no 8.080 , de 19 de setembro de 1990. Dispõe sobre as condições para a promoção, proteção e recuperação da saúde, a organização e o funcionamento dos serviços correspondentes e dá outras providências.

5. Portaria GM/MS nํ569, de $1^{\circ}$ de junho de 2000. Institui o Programa de Humanização no Pré-natal e Nascimento, no âmbito do Sistema Único de Saúde.

6. Portaria GM/MS no 881 - de 19 de junho de 2001. Institui o programa Nacional de Humanização da Assistência Hospitalar/PNHAH.

7. 11a Conferência Nacional de Saúde, Brasília 15 a 19 de dezembro de 2000: o Brasil falando como quer ser tratado: efetivando o SUS: acesso, qualidade e humanização na atenção à saúde com controle social: relatório final / Ministério da Saúde, Conselho Nacional de Saúde. - Brasília: Ministério da Saúde, 2001.

8. Ministério da Saúde. Secretaria-Executiva. Núcleo Técnico da Política

9. Nacional de Humanização. HumanizaSUS: gestão participativa: co-gestão. Brasília:MS, 2004.

10. Ministério da Saúde (MS). Secretaria de Atenção à Saúde. Política Nacional de Humanização da Saúde. Documento Base. $4^{\underline{a}}$ ed. Brasília: Ministério da Saúde (MS); 2007.

11. Ministério da Saúde. Secretaria de Atenção à Saúde. Núcleo Técnico da Política Nacional de Humanização. Grupo de Trabalho de Humanização. 2. ed. 4. reimp. Brasília: MS, 2010. 16 p.

12. Ministério da Saúde. Secretaria de Atenção à Saúde. Núcleo Técnico da Política Nacional de Humanização. HumanizaSUS: documento base para gestores e trabalhadores do SUS. 4. ed. Brasília: MS, 2010a. 72 p.

13. Política Nacional de Humanização, PNH.1aedição, 1areimpressão. Brasília, 2013.

14. Carta dos direitos dos usuários da saúde. Ministério da Saúde. 4. ed. 2013.

15. CAMPOS GWS. Humanização na saúde: Humanização na saúde: um projeto em defesa da vida? Interface Comunic, Saúde, Educação,2005;9(17): 389-406

16. DESLANDES SF. Análise do discurso oficial sobre a humanização da assistência hospitalar. Ciência e Saúde Coletiva, 2004;9(1):7-14.

17. FERREIRA LR, ARTMANN E. Discursos sobre humanização: profissionais e usuários em uma instituição complexa de saúde. Ciência \& Saúde Coletiva, 2018;23(5):1437-1450.

18. FERREIRA ABH. Dicionário Aurélio da Língua Portuguesa. Paraná: Editora Positivo, 2010;2120p.

19. PASCHE DF. Política Nacional de Humanização como aposta na produção coletiva de mudanças nos modos de gerir e cuidar. Interface-Comunicação, Saúde, Educação, 2009; 13(1):701-708.

20. RIOS IC. Humanização: a essência da ação técnica e ética nas práticas de saúde. Revista Brasileira de Educação Médica, v. 33, ano 2, 2009. p. 255-261.

21. SANTOS FSB, et al. A Política Nacional de Humanização como política que se faz no processo de trabalho em saúde. Interface, 2009;13(1):603-613.

22. SILVA JDN et al. Avaliação da satisfação de usuários a partir da Carta SUS, no Rio Grande do Norte, Brasil. Saúde debate [online].2018;42(119):901-915.

23. VAITSMAN J, ANDRADE GRB. Satisfação e responsividade: formas de medir a qualidade e a humanização da assistência à saúde. Ciência e saúde coletiva,2005; 10(3): 599- 613. 